\title{
Malignant Paraganglioma
}

National Cancer Institute

\section{Source}

National Cancer Institute. Malignant Paraganglioma. NCI Thesaurus. Code C8559.

A parag ang lioma that metastasizes to regional or distant anatomic sites. Extraadrenal parag ang liomas have a higher tendency to metastasize, as compared to pheochromocytomas. Common sites of metastasis include the lymph nodes, lungs, bones, and liver. 